\section{New polymer-free dual-drug-eluting stent}

A novel, polymer-free, dual-drug-eluting stent (DES) has shown improved or comparable efficacy to two commercially available, permanent polymer stents: the sirolimus-eluting stent (SES; Cypher ${ }^{\circledR}$, Cordis Corporation, Miami Lakes, FL) and the zotarolimus-eluting stent (ZES; Endeavor ${ }^{\circledast}$, Medtronic Vascular Inc., Santa Rosa, CA). "We demonstrated that the antirestenotic efficacy of the dual-DES was similar to that of the Cypher stent and superior to that of the Endeavor stent," comments Robert Byrne, lead study author.

Durable polymers control the release of active agents from stents; however, the presence of permanent polymer long after its function is useful has been implicated as a potential causal factor in adverse events, such as a persistent inflammatory response at the coronary vessel wall. Byrne and colleagues developed a polymer-free dual (rapamycin and probucol) DES. "Probucol is a potent lipophilic antioxidant that has shown promising antirestenotic efficacy ... [but] has not previously been incorporated in a DES platform," Byrne explains.

The investigators compared the antirestenoic efficacy of the dual-DES with that of the SES-considered the gold standard in terms of antirestenoic efficacy - and the ZES. Patients undergoing coronary stenting were randomly assigned to treatment with a dual-DES $(n=333)$, SES $(n=335)$ or ZES $(n=339)$. Rates of binary angiographic restenosis and target lesion revascularzation in the dual-DES group ( $11.0 \%$ and $6.8 \%$, respectively) were considerably lower than in the ZES group (19.3\% and $13.6 \%)$ and comparable with those observed in the SES group (12.0\% and $7.2 \%$ ).

These data demonstrate that a polymerfree DES can achieve comparable efficacy to stent platforms that use polymers. "The potential safety (and maybe long-term efficacy) advantages of a polymer-free DES

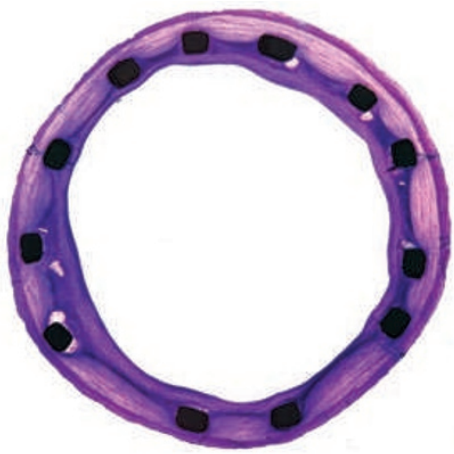

Antirestenotic performance of the rapamycin+probucol-eluting dual-drug-eluting stent in a rabbit model. Permission obtained from M. Joner.

remains putative and subject to extended follow-up in studies involving larger [numbers of patients]," Byrne concludes.

\section{Lisa Richards}

Original article Byrne, R. A. et al. A polymer-free dual drug-eluting stent in patients with coronary artery disease: a randomized trial vs. polymer-based drug-eluting stents. Eur. Heart J. 30, 923-931 (2009). 